\title{
Comparison of Hoechst 33342 and propidium iodide as fluorescent markers for sperm fusion with hamster oocytes
}

\author{
D. P. L. Green \\ Department of Pharmacology, University of Otago Medical School, PO Box 913, Dunedin, \\ New Zealand
}

\begin{abstract}
Summary. Hamster oocytes were loaded with the DNA dyes Hoechst 33342 or propidium iodide. Oocytes incubated in $10 \mu \mathrm{mol}$ Hoechst $333421^{-1}$ showed intracellular fluorescence within $10-20 \mathrm{~s}$ of exposure, as did hamster and guinea-pig spermatozoa. Impaled oocytes to which acrosome-intact hamster spermatozoa were bound before injection of Hoechst 33342 showed dye transfer to adhering spermatozoa within 2 min of injection. Oocytes loaded passively with Hoechst 33342 showed dye transfer to bound, acrosome-intact hamster spermatozoa within $10 \mathrm{~min}$. On ultra. structural examination, no bound, acrosome-intact hamster spermatozoa $(n=311)$ were found to be fused. By contrast, oocytes incubated with $10 \mu \mathrm{mol}$ propidium iodide $1^{-1}$ showed no intracellular fluorescence after $2 \mathrm{~h}$, although in approximately $50 \%$ of oocytes, fluorescence developed rapidly in the first polar body. Oocytes injected with propidium iodide showed intracellular fluorescence but no dye transfer to bound, acrosome-intact hamster spermatozoa. Oocytes impaled on pipettes containing propidium iodide showed no dye transfer to unlabelled oocytes with which they were brought into contact, whereas in similar experiments using Hoechst 33342 detectable dye transfer to an adjacent oocyte occurred within $10 \mathrm{~min}$. Oocytes loaded with propidium iodide transferred propidium iodide to fusion-competent guinea-pig spermatozoa during in vitro fertilization. Normally, between 20 and 40 spermatozoa bound per oocyte, and the percentage of spermatozoa showing dye transfer varied between 0 and $41 \%$. Dye transfer occurred within 5-45 min. Only those nuclei that showed propidium iodide transfer subsequently decondensed, suggesting that dye transfer is correlated with fusion. The presence of fused spermatozoa was confirmed by ultrastructural examination of oocytes. In separate experiments, hamster and guinea-pig spermatozoa showed detectable fluorescence from propidium iodide within $20 \mathrm{~s}$ of osmotic rupture or membrane stripping by detergent, suggesting the lag in dye transfer to sperm nuclei during fertilization reflects a delay in sperm-oocyte fusion following adhesion.

This evidence suggests that Hoechst 33342 could be an unreliable marker for sperm-oocyte fusion in fertilization because of its capacity for passive movement from oocyte to spermatozoon. This problem can be overcome using oocytes injected with propidium iodide. With this technique, it was possible to show that fusion-competent guinea-pig spermatozoa that are held in pipettes will fuse with hamster oocytes when placed mechanically against the oocyte surface.
\end{abstract}

Keywords: spermatozoa; oocyte; cell fusion; fluorescent dye; mammalian fertilization

\section{Introduction}

Hoechst 33342 is a fluorescent DNA dye which was used by Hinkley et al. (1986) to monitor sperm-egg fusion in sea urchin fertilization. It has the advantage that it gives a relatively small 
signal when not bound to DNA, and this makes the fluorescence of a fused spermatozoon easy to detect. Hoechst 33342 has subsequently been used to monitor sperm-oocyte fusion in mammalian fertilization (Conover \& Gwatkin, 1988; Stewart-Savage \& Bavister, 1988; Zuccotti et al., 1991).

Hoechst 33342 is a membrane-permeant dye which passes reversibly into eggs and oocytes following incubation. Passive efflux of Hoechst 33342 from loaded oocytes therefore runs the risk of generating false fusion positives from spermatozoa that bind to, but do not fuse with, the loaded oocyte. In both sea urchin and mouse fertilization, efflux of Hoechst 33342 is slow enough to prevent false positives occurring and only fusions are recorded (Hinkley et al., 1986; Conover \& Gwatkin, 1988). By contrast, hamster oocytes lose Hoechst 33342 rapidly and false fusion positives can be generated (Conover \& Gwatkin, 1988). Zuccotti et al. (1991) overcame the problem by exposing spermatozoa to oocytes loaded with Hoechst 33342 for only $5 \mathrm{~min}$. This time frame would, if obligatory, make it impossible to detect fusions unequivocally where fusion does not occur within $5 \mathrm{~min}$. Methods for detecting fusion optically where fusion is delayed would, nevertheless, be useful because the absence of a barrier to fusion between species makes hamster oocytes useful experimental tools. Experiments were therefore undertaken to re-examine the behaviour of Hoechst 33342 and compare it with the vital DNA dye, propidium iodide, with a view to establishing a time-independent method for monitoring sperm-oocyte fusion optically.

\section{Materials and Methods}

\section{Materials and measurements}

Analytical grade reagents were used throughout. Biological reagents were from Sigma (St Louis, MO, USA), propidium iodide and sulforhodamine B from Molecular Probes (Eugene, OR, USA), and Hoechst 33342 from Serva (Heidelberg, Germany). Osmolalities were measured by vapour pressure osmometry (Wescor, Logan, UT, USA). Guinea-pigs and golden hamsters were supplied by the University's Animal Breeding Station.

\section{Isolation of spermatozoa}

Guinea-pig spermatozoa were flushed from single excised vasa deferentia and caudae epididymides with a modified Tyrode's medium based on that used by Yanagimachi, and called $m$-TAPL (Yanagimachi, 1982). The current medium contains $140 \mathrm{mmol} \mathrm{NaCl1} 1^{-1}$ and $5 \mathrm{mmol} \mathrm{KCl1^{-1 }}$ in place of the $124.8 \mathrm{mmol} \mathrm{NaCl} 1^{-1}$ and $2.7 \mathrm{mmol} \mathrm{KCl}^{-1}$, respectively. The osmolality of the medium used was 300 mosmol $^{-1}$, compared with 266 mosmol $1^{-1}$ for $m$-TAPL. The current medium has been called modified $m$-TAPL. $\mathrm{Ca}^{2+}$-free modified $m$-TAPL contained $2 \cdot 29 \mathrm{mmol}$ $\mathrm{MgCl}_{2} \mathrm{I}^{-1}$ and $100 \mu \mathrm{mol}$ EGTA $\mathrm{l}^{-1}$ in place of $1.8 \mathrm{mmol} \mathrm{CaCl}{ }_{2} 1^{-1}$. Guinea-pig spermatozoa were incubated in modified $m$-TAPL for $18 \mathrm{~h}$ at $37^{\circ} \mathrm{C}$ and were resuspended in fresh medium $\mathrm{l} \mathrm{h}$ before use. Approximately $50-60 \%$ had undergone the acrosome reaction after incubation, and $80-90 \%$ of these spermatozoa were motile. Most of the remaining spermatozoa were clumped, but about $20-30 \%$ of these clumps were also motile. Incubated spermatozoa were prepared for electron microscopy as previously described (Green, 1978). Hamster spermatozoa were obtained by cutting excised caudae epididymides under modified $m$-TAPL (normal or $\mathrm{Ca}^{2+}$-free) and were used immediately.

\section{Isolation and injection of oocytes}

Hamster oocytes were obtained from naturally cycling hamsters on a controlled light-dark cycle. Cumulus masses were removed with hyaluronidase $(0.01 \%)$ and the oocytes washed in modified $m$-TAPL. Oocytes were loaded passively with Hoechst $33342\left(10 \mu \mathrm{mol}^{-1}\right)$ for $10 \mathrm{~min}$, and washed seven times over $15 \mathrm{~min}$. Zonae pellucidae were removed with $0.01 \%$ trypsin at the fifth wash. In parallel experiments with propidium iodide, oocytes were incubated with $10 \mu \mathrm{mol}$ propidium iodide $\mathrm{I}^{-1}$ in modified $m$-TAPL for up to $2 \mathrm{~h}$. The oocytes were pressure (microsurgically) injected in an observation chamber held at $34-36^{\circ} \mathrm{C}$ on a Zeiss IM35 inverted microscope with a Plan $\times 40$ objective (NA 0.65 ) and Nomarski optics. After penetrating the zona pellucida with a pipette, dye was expelled from the pipette for approximately $5 \mathrm{~s}$, the pipette was moved forward and the oocyte itself was then penetrated. The prior expulsion cleared the pipette of diluted dye, and the response of the oocyte and zona pellucida could be used to gauge an appropriate injection pressure. Oocytes were injected immediately after penetration, usually at an injection pressure of 50-100 kPa. The chamber was heated by a water jacket and warm air. Injection pipettes were standard electrode micropipettes pulled on a Brown-Flaming puller (Sutter Instrument Co., San Francisco, CA, USA). Tips were broken to about $1 \lambda$. Injection solutions contained $4.5 \mathrm{mmol}$ propidium iodide $1^{-1}$ or $4.9 \mathrm{mmol}$ Hoechst $33342 \mathrm{I}^{-1}$ in $137 \mathrm{mmol} \mathrm{KCl} 1^{-1}, 2.6 \mathrm{mmol} \mathrm{NaCl} 1^{-1}, 7.8 \mathrm{mmol} \mathrm{Na}_{2} \mathrm{HPO}_{4} 1^{-1}, 1.4 \mathrm{mmol} \mathrm{KH} \mathrm{PO}_{4} 1^{-1}, \mathrm{pH} 7.4$. The final concentration of propidium iodide was estimated to be no more than $360 \mu \mathrm{mol} 1^{-1}$. Oocytes were washed in $\mathrm{Ca}^{2+}$-free 
modified $m$-TAPL immediately before injection, injected with propidium iodide in batches of five in the same medium, and transferred back to modified $m$-TAPL. When $20-30$ oocytes had been injected, zonae pellucidae were removed with $0.01 \%$ trypsin. After washing three times, viable oocytes (i.e. those that showed no detectable swelling) were returned to fresh $m$-TAPL medium in the observation chamber. Alternatively, oocytes were impaled in the chamber, the zona pellucida removed in situ, and the oocyte washed extensively by successive flushing of the observation chamber.

\section{Incubation of oocytes with spermatozoa and their fertilization}

Spermatozoa incubated for $18 \mathrm{~h}$ as described above or zona pellucida-free oocytes were introduced into the observation chamber already holding dye-loaded oocytes. The dye-loaded oocytes were observed using either a Plan $\times 40$ Neofluar (NA 0.9) or a Planapo $\times 63$ (NA 1.4) objective, both oil immersion with Nomarski optics. Fluorophores were excited with a xenon pressure lamp and the following Zeiss filters: PI (ex $493 \mathrm{~nm}$, em $639 \mathrm{~nm}$; $485 \mathrm{~nm}$ band pass, $510 \mathrm{~nm}$ dichroic, $560 \mathrm{~nm}$ long pass (Ditric Optics, Hudson, MA, USA)); Hoechst 33342 (ex $411 \mathrm{~nm}$, em $498 \mathrm{~nm} ; 390-420 \mathrm{~nm}$ band pass, $425 \mathrm{~nm}$ dichroic, $450 \mathrm{~nm}$ long pass); sulforhodamine B (ex $498 \mathrm{~nm}$, em $593 \mathrm{~nm} ; 510-560 \mathrm{~nm}$ band pass, $580 \mathrm{~nm}$ dichroic, $600 \mathrm{~nm}$ long pass). Sulforhodamine B injections were performed separately on additional oocytes to check that injected oocytes did not leak dye rapidly, since this dye is not bound strongly to any intracellular structure or compartment. Oocytes with spermatozoa attached were immobilized before photography to prevent movement of the oocyte during exposure and consequent blurring of images. In the experiments using Hoechst 33342, the exciting light itself was sufficient to immobilize all spermatozoa: for oocytes loaded with propidium iodide, $100 \mu \mathrm{l}$ of $2 \%$ glutaraldehyde in 0.14 mol sodium cacodylate $\mathrm{I}^{-1}, \mathrm{pH} 7 \cdot 2$, was added to the chamber (volume was $2-3 \mathrm{ml}$ when filled). For recording in real-time, the fluorescent image was projected on to a silicon-intensified target video-camera $(\mathrm{RCA} \mathrm{TC1030/H)} \mathrm{and} \mathrm{the} \mathrm{video} \mathrm{image} \mathrm{displayed} \mathrm{on} \mathrm{a} \mathrm{monitor.} \mathrm{The} \mathrm{intensity}$ of the exciting light was adjusted with neutral density filters. However, only propidium iodide had a suitable signal-tonoise ratio for this method to be applicable. Transfer of Hoechst 33342 and propidium iodide could also be detected by eye without difficulty. Fusion of incubated, acrosome-reacted guinea-pig spermatozoa was detected by allowing eggs to proceed to sperm decondensation with simultaneous monitoring of transfer of propidium iodide. Incubated, acrosome-reacted guinea-pig spermatozoa were also placed mechanically against oocytes loaded with propidium iodide. For these experiments, motile, acrosome-reacted guinea-pig spermatozoa were introduced into the observation chamber in small numbers, and a motile spermatozoon sucked by the tail into a patch-clamp pipette the tip of which had been broken to provide an opening of $1-2 \lambda$. Each spermatozoon was placed against a hamster oocyte loaded with propidium iodide and monitored at approximately $5 \mathrm{~min}$ intervals for propidium iodide transfer to the spermatozoon.

\section{Electron microscopy}

Spermatozoa were prepared for electron microscopy as previously described (Green, 1978). Oocytes were fixed for $15 \mathrm{~min}$ in $1 \%$ glutaraldehyde in $0.15 \mathrm{~mol}$ sodium cacodylate $\mathrm{l}^{-1}$ buffer, washed in the same buffer, and transferred to $1 \% \mathrm{OsO}_{4}$ in the same buffer for $2 \mathrm{~h}$. After washing three times in 0.2 mol sodium acetate $1^{-1}$ buffer, $\mathrm{pH} 5 \cdot 1$, oocytes were stained with $1 \%$ uranyl acetate in the same buffer overnight at $4^{\circ} \mathrm{C}$. They were then processed as for spermatozoa (Green, 1978). Sections were examined on a Philips $410 \mathrm{LS}$ electron microscope at $80 \mathrm{kV}$.

\section{Results}

\section{Dye injection}

Injected volumes varied, partly because the size of the pipette tip was not reproducibly the same following breakage, and partly because, where consecutive injections of oocytes were made, the tip could become progressively blocked. In the most successful injections, there was no obvious difference in size between injected and uninjected oocytes (Figs 1-3). The injection volumes were about $10-20 \mathrm{pl}$, or about $4-8 \%$ of cell volume, based on estimates of the increase in cell size. This gives final intracellular concentrations of propidium iodide of about $180-360 \mu \mathrm{mol}^{-1}$. Normally, at least $40 \%$ of a batch of injected oocytes remained stable after injection and withstood trypsin digestion of their zonae pellucidae: on occasion, the percentage was much higher. Swollen oocytes were generally incapable of withstanding removal of the zona pellucida with trypsin. Provided the oocyte was osmotically stable after injection, it made no difference whether the oocyte was impaled or not during zona pellucida removal. 

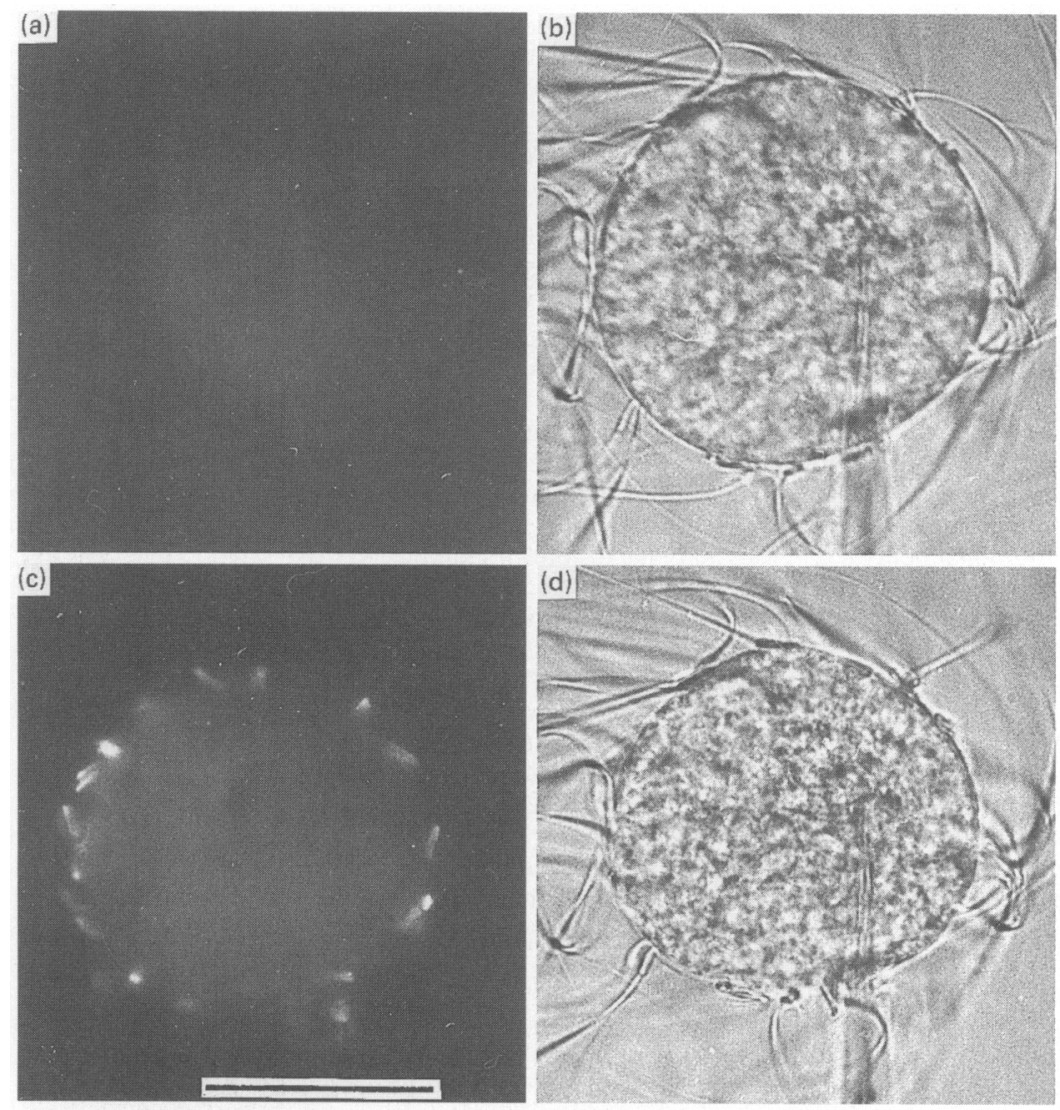

Fig. 1. Hamster oocyte impaled on a pressure-injection pipette containing Hoechst 33342 . The oocyte was impaled and the zona pellucida was then removed. $(a, b)$ Motile, acrosome-intact hamster spermatozoa binding to the oocyte after impalement but before injection. (c, d) The same oocyte $5 \mathrm{~min}$ after injecton of Hoechst 33342. Sperm movement has shifted the oocyte slightly on the pipette. Adhering spermatozoa now fluoresce. It is suggested that dye has diffused passively from the oocyte. Bar $=50 \lambda$.

Dye-injected oocytes fixed after injection $(n=15)$ showed no ultrastructural damage, although some cortical granule discharge was sometimes evident. The potential for dye loss from injected oocytes was examined by loading a number of oocytes separately with the dye sulforhodamine B, which does not bind strongly to cell constituents. In these experiments, $50-60 \%$ of successfully injected oocytes $(n=18)$ retained dye without any obvious reduction in fluorescence for at least an hour.

\section{Exposure of cells to external Hoechst 33342 and propidium iodide}

Hamster oocytes with intact zonae pellucidae showed detectable blue-green fluorescence (about $495 \mathrm{~nm}$ ) in the meiotic spindle and first polar body within 10-20 s of exposure to $10 \mu \mathrm{mol}$ Hoechst $333421^{-1}$, with maximum fluorescence developing within 20-30 s. By contrast, oocytes exposed to $10 \mu \mathrm{mol}$ propidium iodide $\mathrm{l}^{-1}$ showed no intracellular fluorescence following $1-2 \mathrm{~h}$ incubation, although the first polar body in about $50 \%$ of oocytes fluoresced within $30 \mathrm{~s}$ (Fig. 4). Oocytes injected with propidium iodide show fluorescence in both cytoplasm and meiotic spindle (Figs 2 and 3$)$. 

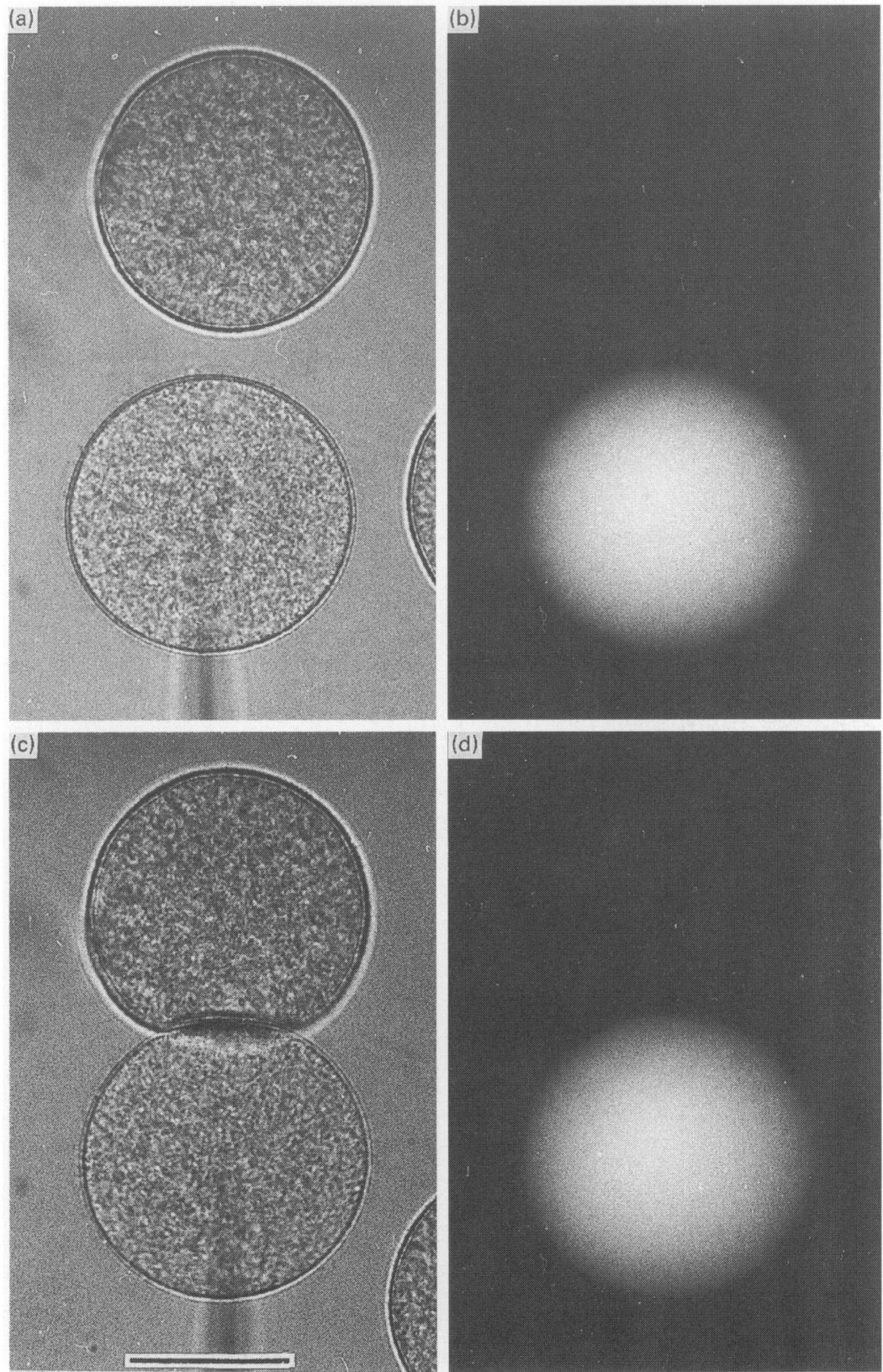

Fig. 2. (a.b) Hamster oocyte after impalement on a pressure-injection pipette containing propidium iodide. This dye causes much greater cytoplasmic fluorescence than Hoechst 33342 . (c, d) The same oocyte after 20 min contact with an unloaded hamster oocyte. No dye transfer is detectable. $\mathrm{Bar}=50 \lambda$.

\section{Dye-loaded oocytes}

The transfer of Hoechst 33342 to bound, acrosome-intact hamster spermatozoa was studied either by impaling oocytes with Hoechst 33342 injection pipettes loaded with Hoechst 33342 (Fig. 1), or by incubating spermatozoa with oocytes preloaded with $\mathrm{H} 33342$ (not shown). In the first experiments, dye transferred progressively to the bound spermatozoa following injection of 

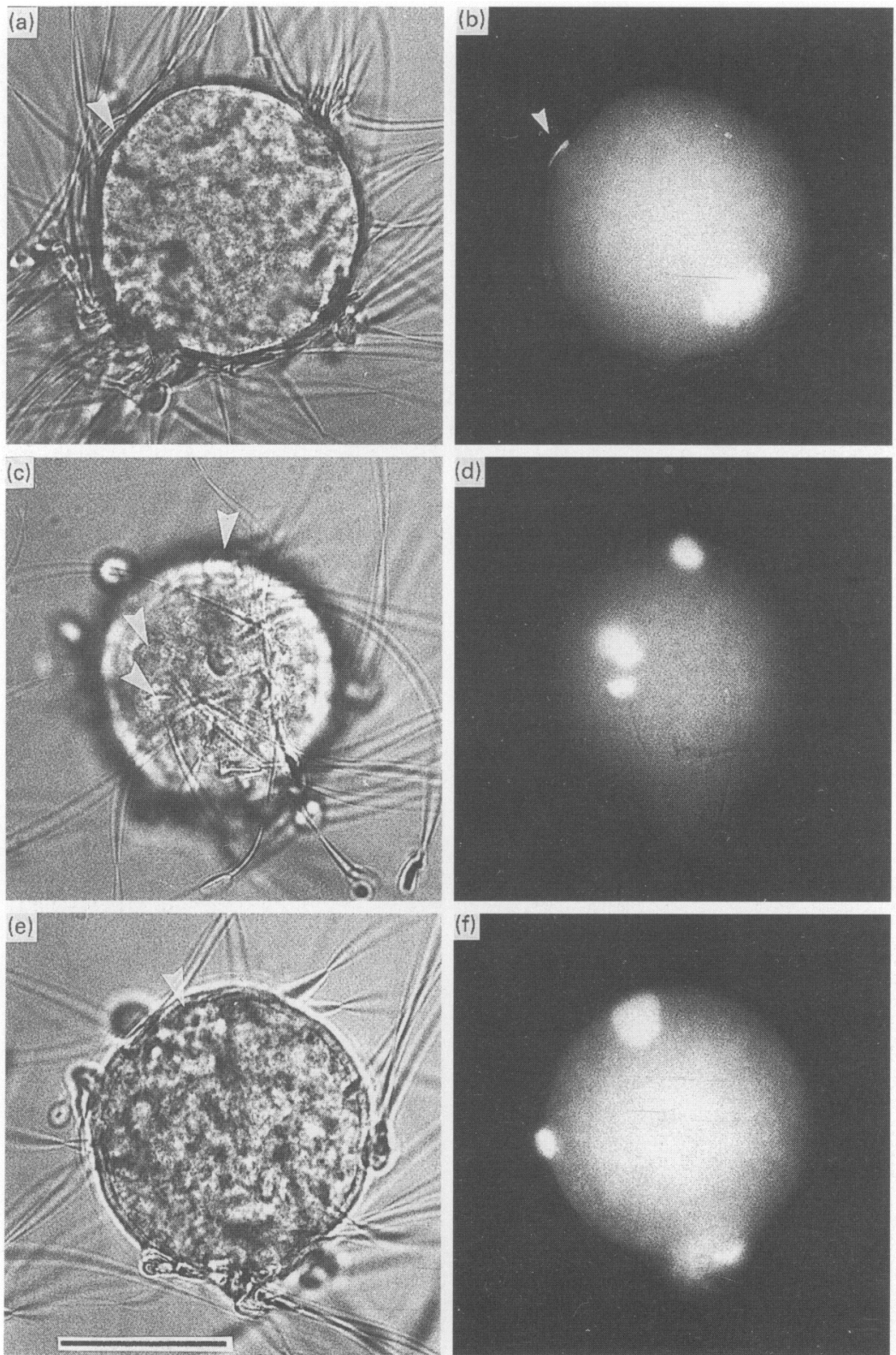

Fig. 3. Hamster oocytes injected with propidium iodide, after fertilization with motile, acrosome-reacted guinea-pig spermatozoa. Oocytes were lightly fixed in glutaraldehyde to immobilize adhering spermatozoa immediately before photography. (a, b) An oocyte 5 min after insemination. Numerous spermatozoa have attached to the surface of the oocyte but only one spermatozoon in this optical plane shows dye transfer (arrow). The position of the spermatozoon on the oocytc surface in (a) can be identified by a slight hummock (arrow). (c, d) An oocyte fixed $75 \mathrm{~min}$ after insemination and focused on one of the poles. Three spermatozoa can be identified in (c) (arrows), which have undergone dye transfer (d). Their nuclei have partly swollen. Ghosts of non-fluorescent spermatozoa can be seen in (d), confirming that prolonged contact with the oocyte is not, by itself, sufficient for dye transfer. (e,f) The same oocyte as in (c, d) but focused in a plane nearer the equator of the oocyte. The upper fluorescent sperm nucleus can be seen in (c) to be considerably more decondensed (arrow) than those in (c). $\operatorname{Bar}=50 \lambda$. 

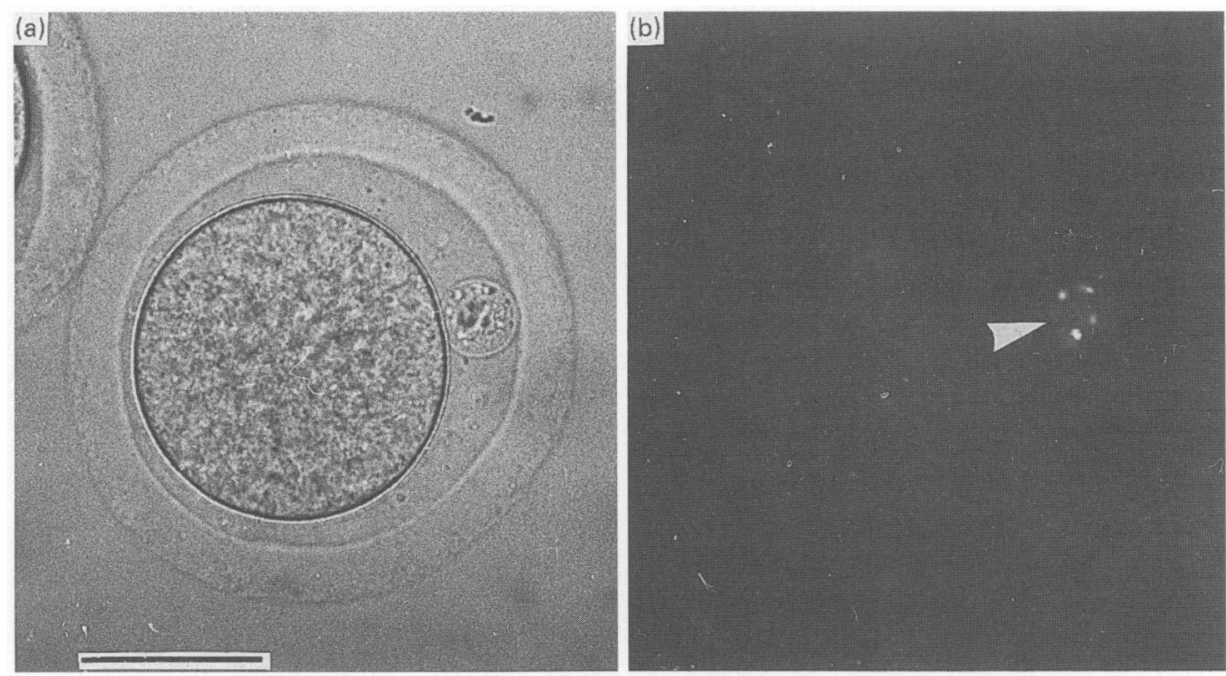

Fig. 4. Hamster oocyte after $1 \mathrm{~h}$ of incubation in $10 \mu \mathrm{mol}$ propidium iodide $\mathrm{l}^{-1}$. (a) Light micrograph showing the position of the first polar body relative to the oocyte. (b) The first polar body fluoresces after 10-20s (arrow), but fluorescence is completely absent from the oocyle itself. Bar $=50 \lambda$.
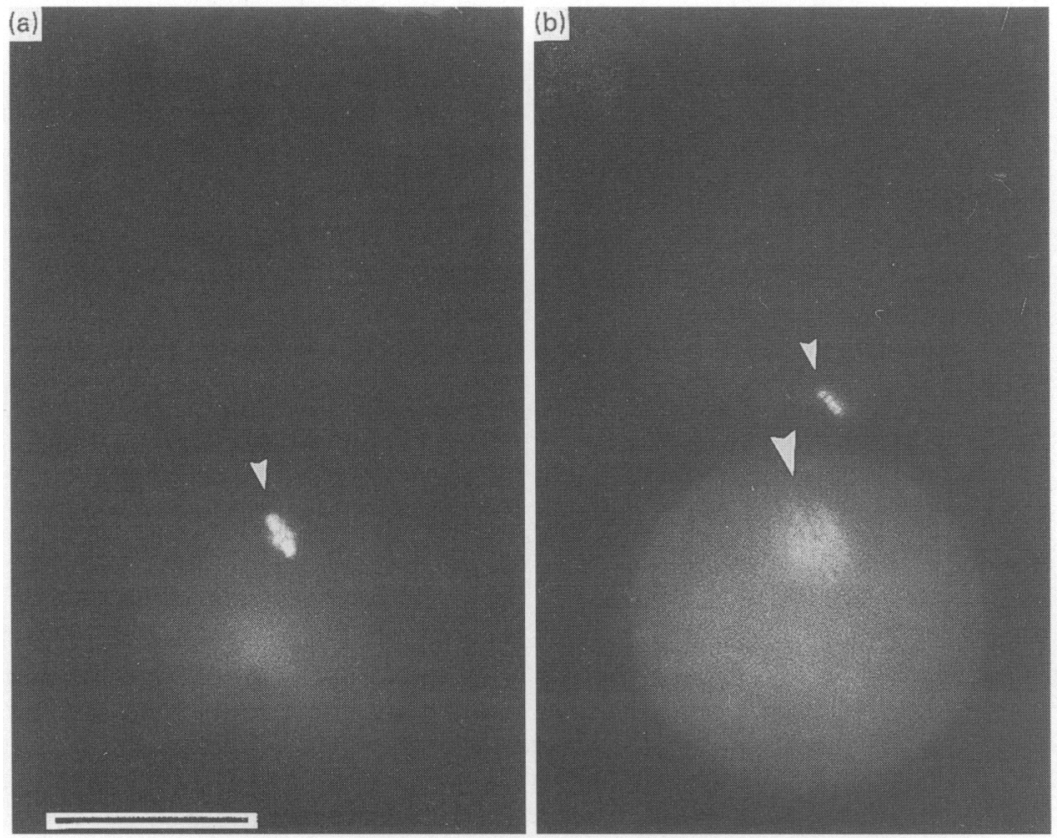

Fig. 5. Hamster oocyte impaled on an injection pipette containing Hoechst 33342 and placed against an unloaded oocyte as in Fig. 2. (a) The impaled oocyte $10 \mathrm{~s}$ after injection. The meiotic spindle is fluorescent (arrow). (b) The same oocytes 10 min later. The cytoplasm of the impaled oocyle is more fluorescent and dye has transferred to the non-impaled oocyte. causing its meiotic spindle to fluoresce (small arrow). The plane of focus is slightly different from that in (a) to show this spindle better. but the spindle in the impaled oocyte is still visible (large arrow). 

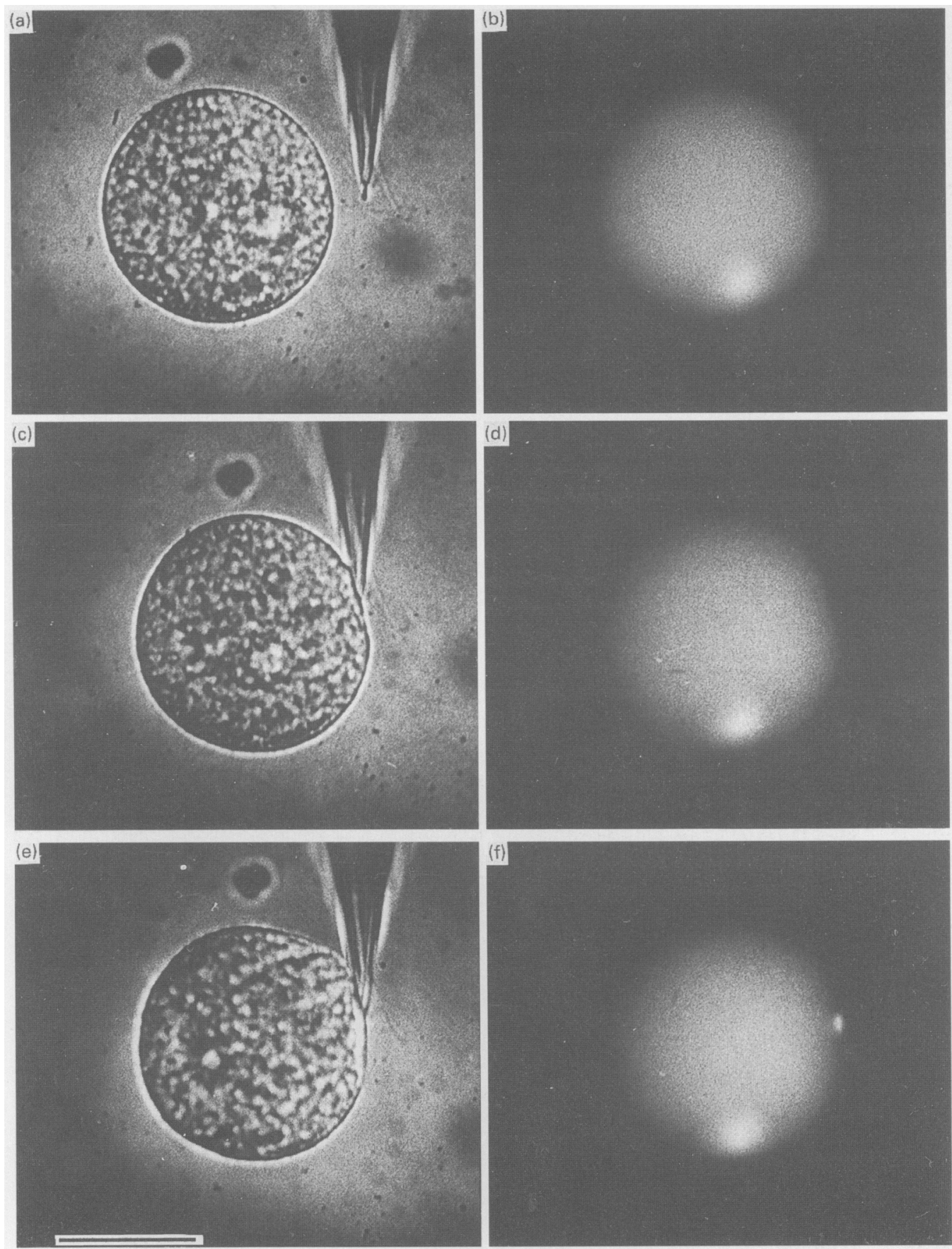

Fig. 6. Hamster oocyte injected with propidium iodide, against which a fusion-competent. acrosome-reacted guinca-pig spermatozoon has been placed with a pipette. (a) A micro-pipette has a spermatozoon drawn into it by its tail. The sperm head is vertical to the plane of the micrograph and appears as a small linear projection. The oocyte has been loaded with propidium iodide and the injection pipette withdrawn. (b) The propidium iodide in the oocyte fuoresces. (c) The oocyte is pushed against the spermatozoon. (d) Fluorescence micrograph of (c) showing that there is initially no dye transfer to the spermatozoon from the oocyte. (e, f) Micrographs taken approximately $25 \mathrm{~min}$ after (c, d). The sperm nucleus now fluoresces. suggesting that fusion has occurred. The images were obtained using a SIT camera, and photographed directly from a TV monitor. $\operatorname{Bar}=50 \lambda$. 
Hoechst 33342 (Fig. 1). In the second experiments, epididymal hamster spermatozoa bound to oocytes loaded with Hoechst 33342 showed dye transfer to adhering spermatozoa within 10 min of binding. Oocytes loaded by incubation with Hoechst 33342 and fixed after incubation with hamster spermatozoa showed no evidence of sperm fusion (311 spermatozoa) when examined by electron microscopy. Similar experiments with oocytes loaded with propidium iodide showed no dye transfer either to bound hamster spermatozoa (not shown), or to hamster oocytes brought into contact with an impaled oocyte (Fig. 2). Similar experiments with oocytes impaled on pipettes loaded with Hoechst 33342 showed detectable dye transfer to an adjacent oocyte within $10 \mathrm{~min}$ (Fig. 5a, b).

\section{Dye transfer from oocytes to spermatozoa following fusion}

Spermatozoa must undergo the acrosome reaction under conditions that preserve their motility if they are to become fusion competent and fertilization is to occur in vitro. Unfortunately, spermatozoa from the single strain of hamsters available in New Zealand do not undergo the acrosome reaction under conditions that normally induce the acrosome reaction and render hamster spermatozoa fusigenic. Moreover, the acrosome reaction can be induced only with the ionophore A23187 in 10-20\% of spermatozoa, and these spermatozoa lose their motility. Since hamster colonies are tightly regulated here, and the cost of introducing new strains into the country is prohibitively high, capacitated guinea-pig spermatozoa were used instead (Yanagimachi, 1972). Motile, acrosome-reacted guinea-pig spermatozoa bind to the plasma membrane of oocytes loaded with propidium iodide, and dye transfer normally occurs 5-45 minutes later (Fig. 3). On some occasions, no spermatozoa showed dye transfer; on another, the number was as high as 17 . As the number of spermatozoa that bind is normally about $20-40$, the percentage showing dye transfer lies between 0 and about $41 \%$ of those bound. As yet, there appears to be no experimental variable that correlates with the number of spermatozoa to which dye is transferred by any one oocyte. Some spermatozoa adhere and continue to move vigorously for an hour or two but never show dye transfer. Other spermatozoa, which move more sluggishly after adhesion, may fuse after $20 \mathrm{~min}$. Those nuclei showing propidium iodide transfer subsequently decondense (Fig. 3c-f), whereas those that do not show dye transfer do not decondense. The intensity of fluorescence in the nucleus can fall with decondensation. In experiments in which pre-incubated, pipette-held spermatozoa were brought into contact with oocytes loaded with propidium iodide $(n=8)$, fusion occurred on three occasions (Fig. 6). These fusions took place between 15 and 35 min of placement.

\section{Propidium iodide uptake by spermatozoa}

Hamster and guinea-pig spermatozoa suspended in $10 \mu \mathrm{mol}$ propidium iodide $\mathrm{l}^{-1}$ do not fluoresce if they are motile, but immotile spermatozoa often show fluorescence of the head. When spermatozoa are subject to osmotic shock or detergent, fluorescence develops in the head within $20 \mathrm{~s}$. Motile spermatozoa, incubated with $10 \mu \mathrm{mol}$ Hoechst $33342 \mathrm{l}^{-1}$, also show fluorescence in the head after $10-20 \mathrm{~s}$.

\section{Discussion}

Hoechst 33342 and propidium iodide are dyes that undergo enhancement of fluorescence when bound to DNA. Hoechst 33342 is membrane permeant, although its uptake is dependent on temperature, external concentration and cell type. Evidence for the membrane permeancy of Hoechst 33342 is extensive, and includes the evidence that it can be loaded into sea urchin eggs and 
hamster and mouse oocytes merely by incubation (Hinkley et al., 1986; Conover \& Gwatkin, 1988; Stewart-Savage \& Bavister, 1988; Zuccotti et al., 1991). The evidence presented here indicates that Hoechst 33342 enters hamster oocytes rapidly. Propidium iodide, by contrast, is a vital stain (Krishnan, 1975; Ciancio et al., 1988), and passive movement into hamster oocytes is below the limit of detection.

This difference between the two dyes has important practical implications for their use as markers of sperm-oocyte fusion. Whereas oocytes can be expected to retain propidium iodide following injection, provided the cells seal properly after withdrawal of the injection pipette, they cannot necessarily be expected to retain Hoechst 33342 after passive loading. It is difficult to be certain how fast Hoechst 33342 leaks out of loaded hamster oocytes, since the only indicator of its presence is the fluorescence of the meiotic spindle. There is no evidence that Hoechst 33342 bound to DNA equilibrates rapidly with the cytoplasmic pool when this pool empties, and the intensity of fluorescence in the spindle cannot be taken as an indicator of cytoplasmic concentration. Whatever the rate of loss, oocytes will undoubtedly contain a declining reservoir of Hoechst 33342 for transfer to spermatozoa in the event of cell fusion, and are a source of dye for passive uptake by cells in the vicinity of their surfaces. Evidence suggests that acrosome-intact hamster spermatozoa, which adhere to hamster oocytes but do not fuse with them, do take up dye passively from oocytes loaded with Hoechst 33342 (Conover \& Gwatkin, 1988; Zuccotti et al., 1991). However, bound, acrosome-intact hamster spermatozoa fail to take up dye from oocytes injected with propidium iodide, and so do other oocytes. Electron micrographs show no evidence of fusion between acrosome-intact hamster spermatozoa and oocytes (data not shown; see also Yanagimachi \& Noda (1970)), nor between oocytes. The only reasonable conclusion is that Hoechst 33342 can transfer to bound, but unfused, spermatozoa, whereas propidium iodide cannot.

The view that propidium iodide is membrane impermeant in oocytes in reinforced by the response of motile, acrosome-reacted (i.e. incubated) guinea-pig spermatozoa (Yanagimachi, 1972) bound to cells loaded with propidium iodide. Those spermatozoa that show dye transfer subsequently undergo decondensation of their nuclei, indicating fusion and fertilization, whereas those that do not show dye transfer show no decondensation either. Electron microscopy confirms the presence of fused spermatozoa in oocytes showing dye transfer. Given the time lag between injection of oocytes with propidium iodide and the uptake of dye by fusing spermatozoa, it is clear that oocytes loaded with propidium iodide can retain dye for extended periods. Since damaged spermatozoa fluoresce rapidly after exposure to external propidium iodide, dye transfer from oocyte to spermatozoa is likely to reflect a rapid equilibration of cytoplasmic pools after fusion. On this basis, the delay between adhesion of capacitated spermatozoa and their subsequent fluorescence indicates a latency before fusion occurs. Although the matter was not studied in detail, it is clear that hamster oocytes without their zonae pellucidae can become markedly polyspermic.

The retention of propidium iodide by injected oocytes permits study of fusion where the attachment or fusion of spermatozoa is delayed; for example, where fusion-competent guinea-pig spermatozoa are applied mechanically to the oocyte surface. Immotility per se does not appear to prevent fusion. However, the force with which the sperm-oocyte contact is made is unknown. Comparison of deformations of pipette-held spermatozoa with those produced by swimming spermatozoa suggests that the force of mechanical placement is higher. Whether this compressive force increases the likelihood of fusion by deforming the oocyte surface and increasing the area of contact is unknown at present, but clearly worth investigating further. Earlier work on the relation of motility to fusigenicity produced contradictory conclusions (Aitken et al., 1983; Barg et al., 1986). However, where immotility produced an inability to fuse (Barg et al., 1986), the immotility was caused by using the ionophore A23187 to induce the acrosome reaction. Since it is still unclear precisely how spermatozoa are rendered fusigenic by prolonged incubation (as here), it may be inappropriate to connect an absence of fusion with immotility. The preliminary data cited in this paper suggest that the acquisition of fusigenicity by spermatozoa may be a more complex process than simply undergoing an acrosome reaction, and that motility per se is not needed for fusion to 
occur. Clearly, however, the motility of spermatozoa may help spermatozoa to increase contact between themselves (particularly their equatorial segment) and the oocyte surface, something that direct mechanical application of spermatozoa may do simply by deforming the oocyte cortex.

This work was supported by grants from the New Zealand Medical Research Council and the New Zealand Lottery Grants Board.

\section{References}

Aitken, J.R., Ross, A. \& Lees, M.M. (1983) Analysis of sperm function in Kartagener's syndrome. Fertility and Sterility 40, 696-698.

Barg, P.E., Wahrman, M.Z., Talansky, B.E. \& Gordon, J.W. (1986) Capacitated, acrosome reacted but immotile sperm, when microinjected under the mouse zona pellucida, will not fertilize the oocyte. Journal of Experimental Zoology 237, 265-274.

Ciancio, G., Pollack, A., Taupier, M.A., Block, N.L. \& Irvin III, G.L. (1988) Measurement of cell-cycle phase-specific cell death using Hoechst 33342 and propidium iodide: preservation by ethanol fixation. Journal of Histochemistry and Cytochemistry 36, 1147-1152.

Conover, J.C. \& Gwatkin, R.B.L. (1988) Pre-loading of mouse oocytes with DNA-specific fluorochrome (Hoechst 33342) permits rapid detection of spermoocyte fusion. Journal of Reproduction and Fertility 82, 681-690.

Green, D.P.L. (1978) The induction of the acrosome reaction in guinea-pig sperm by the divalent metal cation ionophore A23187. Journal of Cell Science 32, 137-151.

Hinkley, R.E., Wright, B.D. \& Lynn, J.W. (1986) Rapid visual detection of sperm - egg fusion using the DNAspecific fluorochrome Hoechst 33342. Developmental Biology 118, 148-154.
Krishnan, A. (1975) Rapid flow cytofluorometric analysis of mammalian cell cycle by propidium iodide staining. Journal of Cell Biology 66, 188-193.

Stewart-Savage, J. \& Bavister, B.D. (1988) A cell surface block to polyspermy occurs in golden hamster eggs. Developmental Biology 128, 150-157.

Yanagimachi, R. (1972) Penetration of guinea-pig spermatozoa into hamster eggs in vitro. Journal of Reproduction and Fertility 28, 477-480.

Yanagimachi, R. (1982) In vitro sperm capacitation and fertilization of golden-hamster eggs in a chemically defined medium. In In Vitro Feritizaton and Embryo Transfer, pp. 65-76. Ed. E. S. E. Hafez and K. Semm. MTP Press, Lancaster.

Yanagimachi, R. \& Noda, Y.D. (1970) Physiological changes in the postnuclear cap region of mammalian spermatozoa: a necessary preliminary to the membrane fusion between sperm and egg cells. Journal of Ultrastructure Research 31, 486-493.

Zuccotti, M., Yanagimachi, R. \& Yanagimachi, H. (1991) The ability of hamster oolemma to fuse with spermatozoa: its acquisition during oogenesis and loss after fertilization. Development 112, 143-152.

Received 30 September 1991 